\title{
Model of microwave effects on bacterial spores
}

Dmitry Malyshev ${ }^{*}$, Catrin F. Williams², Jonathan Lees², Les Baillie ${ }^{1}$, Adrian Porch ${ }^{2}$

${ }^{1}$ School of Pharmacy and Pharmaceutical Sciences, Cardiff University, Redwood Building, King Edward VII Avenue, Cardiff, CF10 3NB, United Kingdom

${ }^{2}$ School of Engineering, Cardiff University, Queen's Buildings, The Parade, Cardiff, CF24 3AA, United Kingdom

*Correspondence

Dmitry Malyshev

malyshevd@cardiff.ac.uk

Keywords: spores, SEM, electric field, RF, microwaves 


\begin{abstract}
A recent application of microwaves in the healthcare area is for the rapid detection of bacterial spores, particularly of clinically significant spores such as Clostridium difficile. Here, we present a working model of $C$. difficile spore disruption by the action of a $2.45 \mathrm{GHz}$ microwave electric field, independent of overall sample heating. The model shows how inner layers of the spore with the lower complex permittivity values will be subject to higher values of electric field. The model also shows how the electric effects can be enhanced, focusing the electric field into "hotspots" using 'angled' nanoparticles, yielding effective DNA release even at low microwave power levels. The model's predictions have been validated through experimental results, which show an enhancement of DNA release from spores.
\end{abstract}




\section{Introduction}

Infections linked to Clostridium difficile are a significant problem. In hospitals, the organism is primarily acquired through the faecal-oral route as spores excreted by infected patients contaminate the healthcare environment. Data from Public Health England (PHE C. difficile annual data 2015) and Wales (PHW monthly C. difficile update 2015) and the Office of

National Statistics (ONS C. difficile 2015) show that there were approximately 15,000 confirmed cases in England and 2,000 in Wales in 2012, with a total of around 2,000 reported deaths in England and Wales combined.

When under stress, low on nutrients or following oxygen exposure, C. difficile vegetative cells sporulate (i.e. form spores) (Đapa et al., 2013). Spores are resistant to antibiotics, stomach acid, desiccation, temperatures of up to $95^{\circ} \mathrm{C}$, alcohol-based cleaning agents and other chemical biocides, and can survive for several months on surfaces and within the environment (Fordtran, 2006, Kim et al., 1981).

Existing methods to detect the presence of $C$. difficile spores in clinical samples are slow and expensive. Tests such as the cell cytotoxicity assay can take up to two days and are expensive due to the tissue culture requirement. PCR (polymerase chain reaction) assays such as Cepheid Xpert can give detection in 45 minutes from a clinical sample, but are labour intensive (Barbut et al., 2009). In addition, the problem with DNA assays is the requirement for DNA to be readily presentable. Without extracting the DNA, these assays will not work for testing surfaces or early stages of infection as the DNA will be 'locked' within the spores and so will be inaccessible for PCR.

To counteract the issues associated with diagnosis, a sensor which uses microwave extraction of DNA is currently under development, which can detect pathogens in under one minute. The technology was originally based on microwave-accelerated metal-enhanced fluorescence (MAMEF, Aslan et al., 2008, 2010) and was shown to work on both Bacillus and Clostridium spores (Joshi et al., 2014). This technology is being developed further to incorporate the use of 
a bespoke microwave cavity resonant at a frequency of $2.45 \mathrm{GHz}$, that focuses the electric field component into the sample volume, with the goal of using added nanoparticles to further intensity the electric field and so reduce the microwave power requirements of the system. However, to fully understand the role of nanoparticles and the underlying mechanisms at play in the extraction process, it is necessary to model the interaction of bacterial spores with the microwave fields.

Microwaves have been used since the 1990s to deactivate bacteria and spores, for both hospital applications (Pellerin, 1994) and industrial food treatment (Vaid and Bishop, 1998). Microwaves have also been used to inactivate both vegetative bacteria and spores, including Bacillus anthracis, Bacillus subtilis (Celandroni et al. 2004) and Bacilllus licheformus (Kim et al., 2009).

Typically, the microwave mechanism of action in biological applications is assumed to be heating effects alone. Non-thermal effects were reported by Celandroni et al. (2004), but no mechanism was suggested. In order to fully appreciate the contribution of non-thermal effects, it is necessary to consider the spore structure at the molecular level.

A spore contains polar molecules (such as polar groups on proteins) within the tightly packed spore coat. The movement of the polar regions in response to the alternating microwave electric field can cause structural damage to the coat, without any requirement for heating. These effects were observed in high power microwave exposure by Celandroni et al. (2004), whereby B. subtilis spores exhibited structural changes that appeared to be different to the effects caused by direct heating by traditional means. Similar results were later obtained by Kim et al., (2009) with $B$. licheniformis. Therefore, high power microwave exposure may cause spore damage, distinct to that of heating.

The inclusion of nanoparticles has been reported to result in the physical breaking of the bacterial cell walls, and that this effect is related to the shape of the nanoparticles (Acharya et al., 2018). Following on from this, it is possible that different shaped nanoparticles will have distinct effects on spores and may be able to physically damage the spore outer surface. To investigate this, spherical, angled "urchin" shaped nanoparticles were investigated alongside spherical nanoparticles to test if the enhanced electric field around expected to develop around angled nanoparticles results in damage to the spore surface. 
In the work of Aslan et al. (2008), silver nanoparticles in the form of silver island films were used in conjunction with microwaves to disrupt B. anthracis spores. However, these were only used as substrates for fluorophores to which the DNA would bind. The authors hypothesised that the DNA moves towards the nanoparticles, driven by the temperature gradient, with silver being colder due to it not being heated directly by microwaves. More recently, $70 \mathrm{~nm}$ magnetite $\left(\mathrm{Fe}_{2} \mathrm{O}_{3}\right)$ nanoparticles were used as an in vitro demonstration of localised hyperthermia of biological tissue for cancer treatment (Kim et al., 2016). In this experiment, the nanoparticletreated sample reached a higher overall temperature compared with untreated tissue. The nanoparticles themselves do not heat significantly, but enhance the heating of tissue (Pearce $e t$ al., 2016).

Our work has a unique approach in that the goal is to use the nanoparticles to directly interact with the spores, enhancing the disruptive effect of microwaves for DNA release. To understand how the nanoparticles and spores interact under an applied microwave electric field, it was necessary to model these interactions and then verify experimentally that the results correlate with the model's predictions.

\section{Methods}

\subsection{Microwave system components}

The arrangement of the main components which form the microwave exposure system is described below. The microwave signal generator (Telemakus TEG-4000-1) was connected to an RF switch (Telemakus TES-7000-30), which using a bi-directional coupler (Mini-Circuits ZABDC20-322H-S+) drives a power amplifier (Mini-Circuits ZHL-30W-252-S+). The output of the power amplifier was connected, via another directional coupler (Mini-Circuits ZABDC20), to a cylindrical resonant $\mathrm{TM}_{010}$ cavity, previously described by Williams et al., 2015, resonant at $2.45 \mathrm{GHz}$. The coupled forward and reflected signals were measured using two power sensors (Telemakus TED-8000-40).

The generated signal passed through an RF switch, where, depending on the switch state, it is either directed to the power amplifier, or to the coupled port of a bi-directional coupler, where

it is attenuated by approximately $20 \mathrm{~dB}$. The power amplifier increases the signal power by approximately $+47 \mathrm{~dB}$ (from $1 \mathrm{~mW}$ up to $50 \mathrm{~W}$ rms), and this signal then enters the cavity. A second bi-directional coupler allows measurement of the signal power. The circuit schematic is shown in Figure 1. 
Figure 1. A schematic representation of the microwave power delivery system, consisting of source, amplifier and applicator (cavity). The theoretical full power output, after cable and equipment losses have been taken into account, is approximately $+45 \mathrm{dBm}(31.6 \mathrm{~W})$.

The system was operated from a laptop computer connected to all instruments by USB. A custom program (written in National Instruments LabVIEW 2012) was responsible for system control and providing the user interface.

\subsection{Modelling of Nanoparticles and Spores}

A physical model for nanoparticle focusing of the microwave electric field was built in COMSOL Multiphysics 5.0. Spherical nanoparticles of diameter $100 \mathrm{~nm}$ were centred in a $1000 \mathrm{~nm}$ cube of deionised water. The relative permittivity of water was set as 80 at a microwave frequency of $2.45 \mathrm{GHz}$. A uniform electric field was applied parallel to the z-axis, penetrating the whole water cube. In this geometry we expect the usual polar enhancement of the electric field magnitude around a spherical metal particle by a factor of 3 over that of the applied field.

In a similar manner, a spore was simulated as a four-layer ellipsoid with the layers representing the exosporium, coat, membrane and core of the spore. The relative permittivity of the layers were set as follows: 40 for the exosporium and the core, 10 for the coat, and 2.5 for the membrane. The lower permittivity of membranes is discussed in the literature but the values vary depending on composition (Dilger at al., 1979; Tian, 2010). The exact values for spore membranes have not been measured, and the exact composition of the spore membrane, coat and exosporium are not sufficiently known to allow phantoms to be made, hence values were extrapolated from similar materials. Note that the relative permittivity of biological material at microwave frequencies is usually considered to be a complex quantity $\varepsilon=\varepsilon_{1}-\mathrm{j} \varepsilon_{2}$, with the real part quantifying polarisation and the imaginary part quantifying energy loss. For all materials we will assume that $\varepsilon_{1}>\varepsilon_{2}$ (as is the case for water and many biological materials at microwave frequencies around $2.45 \mathrm{GHz}$ ), so for simplicity we will only consider the real part $\varepsilon_{1}$ in our simulations.

Table 1 . Assumed relative permittivity $\varepsilon_{1}$ values

\begin{tabular}{|l|l|l|}
\hline Layer & $\varepsilon_{1}$ & Source for value extrapolation \\
\hline
\end{tabular}




\begin{tabular}{|l|l|l|}
\hline Exosporium & 40 & Lyu et. al., 2018 \\
\hline Coat & 10 & Bibi et. al., 2016 \\
\hline Membrane & 2.5 & Dilger at al., 1979, Tian, 2010 \\
\hline Core & 40 & Lyu et. al., 2018 \\
\hline
\end{tabular}

The spore itself was modelled as an ellipsoid with semiaxis dimensions of 1.0, 0.7 and $0.7 \mu \mathrm{m}$, in accordance with typical spore geometries reported (Paredes-Sabja et al., 2016). Layer thicknesses within the membranes of $C$. difficile spores vary significantly both between different strains and within a single strain, so a typical layer thickness of $0.1 \mu \mathrm{m}$ was chosen for all the layers; this is representative with values observed experimentally, as shown in Figure 2.

Figure 2. Transmission electron micrographs of $C$. difficile (adapted from Paredes-Sabja et al., 2012) highlighting the variation in layer thicknesses and morphologies within the same strain.

\subsection{Preparing purified spore suspensions}

Spores of four isolates of $C$. difficile were used (National Anaerobic Reference Unit, Cardiff, Wales). The CD630 strain was chosen due to being fully genome sequenced (Lawley et al., 2009). The R20291 and DS1813 strain were chosen due to belonging to the 027 "hypervirulent" ribotype. The DS1748 strain was chosen due to reported difference in hydrophobicity from other strains (Joshi et al., 2012).

The method was adapted from that of Sorg and Sohenshein (Sorg and Sohenshein, 2008;, 2010). Bacteria were incubated anaerobically in BHIS (brain-heart infusion) agar at $37^{\circ} \mathrm{C}$ for 4 days. Following incubation, cells were collected from the surface of the plate, using a $10 \mu \mathrm{l}$ inoculating loop and suspended in $1 \mathrm{ml} \mathrm{SDW}$ (sterile deionised water) in a sterile Eppendorf tube. The suspension was incubated at $4^{\circ} \mathrm{C}$ overnight and then centrifuged at $5000 \mathrm{~g}$ for 5 minutes, after which the supernatant was discarded and the pellet was re-suspended in $1 \mathrm{ml}$ of ice cold water. This washing step was repeated 4 times. The suspensions were then layered onto a $50 \%$ sucrose solution and centrifuged at 3,400 g for 20 minutes, after which the pellet was re-suspended in $1 \mathrm{ml}$ of ice cold water. The suspension was then centrifuged at $5000 \mathrm{~g}$ for 
5 minutes and the pellet was re-suspended in $1 \mathrm{ml}$ of ice cold water. This washing step was also repeated 4 times. The resulting final pellet was re-suspended in SDW and stored at $4^{\circ} \mathrm{C}$.

\subsection{DNAse treatment of spores}

Purified spores were further treated to remove any DNA from solution and spore surfaces. DNAse treatment was adapted from Herman et al. (1995). A sample of $40 \mathrm{ng}$ of DNase I in $0.067 \mathrm{M}$ phosphate buffer $(\mathrm{pH} 7)$ and $15 \mathrm{mM} \mathrm{MgCl} 2$ was incubated at $37^{\circ} \mathrm{C}$ with a spore sample of $1 \mathrm{ml}$ in volume, containing $10^{8}$ spores, with aliquots taken every 24 hours. The concentration of DNA in each aliquot was measured using Qubit dsDNA and ssDNA detection, as detailed in section 2.5 .2 below.

For the samples to be stored as stocks for later experiments, the DNAse was deactivated by heating the sample up to $70^{\circ} \mathrm{C}$ for $15 \mathrm{~min}$. The samples were washed by centrifuging at 5000 $\mathrm{g}$ and the samples pellets were re-suspended in SDW.

\subsection{Validation Experiments}

Spores were exposed to microwaves (in this case within the strong microwave electric field region along the axis of the cavity) using the cylindrical microwave cavity described by Williams et al., 2015, made wholly of aluminium. A $170 \mu \mathrm{l}$ sample, at a concentration of $10^{7}$ spores $/ \mathrm{ml}$ for spore samples, was placed in a $200 \mu \mathrm{l}$ capped Eppendorf tube. The Eppendorf was then placed on the axis of the cavity via its axial sample hole. The response of the cavity with the sample was assessed using a network analyser (Agilent N1996A), whereby the microwave frequency was carefully adjusted to equal the frequency of the minimum of the voltage reflection coefficient. After final tuning of the cavity, to ensure an impedance match to its input circuity (for maximum power transfer), the sample was exposed to the microwave electric field, with duration, power and duty cycle all pre-selected.

\subsection{Nanoparticles used}

The nanoparticles chosen for this study, and the key reason behind their choice, are shown in Table 1. With the exception of the germanium nanoparticles, which were sourced from US Nano, all other nanoparticle suspensions were obtained from Sigma-Aldrich. The nanoparticle suspensions from Sigma-Aldrich were supplied as a dispersion in citrate buffer at a concentration of $3.8 \times 10^{9}$ particles $/ \mathrm{ml}$. The germanium nanoparticles came with their own 
proprietary surfactant (formula not provided) and were mixed to a concentration $3.8 \times 10^{9}$ particles $/ \mathrm{ml}$.

Table 2. Nanoparticle types used

\begin{tabular}{|l|l|}
\hline Nanoparticle & Effect being tested \\
\hline $100 \mathrm{~nm}$ gold spherical & Spherical nanoparticle field focusing \\
\hline $30 \mathrm{~nm}$ gold spherical & The effect of smaller size particles \\
\hline $90 \mathrm{~nm}$ gold nano-urchins & $\begin{array}{l}\text { Particles with a spheroid shape with many pointed } \\
\text { protrusions }\end{array}$ \\
\hline $30 \mathrm{~nm}$ germanium & Thermal effect of the nanoparticles + field focusing \\
\hline
\end{tabular}

A $10 \mu \mathrm{l}$ aliquot of the spores was taken, first purified via the Sorg method, as previously described, and DNAse-treated (concentration of $10^{8}$ spores $/ \mathrm{ml}$ ). This aliquot was mixed with $170 \mu \mathrm{l}$ of nanoparticle suspension $\left(3.8 \times 10^{9} \mathrm{particles} / \mathrm{ml}\right)$. A $10 \mu \mathrm{l}$ aliquot was taken from this mixture to serve as the non-microwaved control sample. The remaining $170 \mu \mathrm{l}$ was loaded into the microwave cavity as previously described, using a $200 \mu$ Eppendorf tube. In separate experiments on empty tubes, the plastic material of the tube was found to be of low permittivity, very low microwave loss, and so has very little interaction with the microwave electric field. The samples were exposed to microwave electric field using a $12 \mathrm{~W}, 0.3 \%$ duty cycle (i.e. $3 \mathrm{~ms}$ "ON" time per $1000 \mathrm{~ms}$ ) for 30 seconds. The ssDNA content of the microwaved and nonmicrowaved (control) samples were then quantified using the Qubit system.

Statistical analysis of the results was carried out in Graphpad Prism 5.0. Two-way ANOVA was used to determine the overall significance of results, while Bonferroni tests were used to compare different data sets to the controls.

\section{Results}

\subsection{Estimating nanoparticle heating}

\subsubsection{Particle heating basic model}

The first step in modelling the nanoparticle interactions was to understand how the nanoparticles heat over time when subjected to constant power dissipation per unit volume. Heating of a material (i.e. change in temperature) over time can be described by the following simple equation: 


$$
\frac{d T}{d t}=\frac{Q}{\sigma m}
$$

where $\mathrm{Q}$ is the net heating power, $\sigma$ is the heat capacity of the material and $m$ is the mass of the material. We can expand Q into:

$$
\begin{aligned}
Q & =P-\alpha A \Delta T \\
& =P-\alpha A\left(T_{S}-T_{W}\right)
\end{aligned}
$$

where $\mathrm{P}$ is the power dissipated (in our case, power absorbed) by the nanoparticles when exposed to microwaves, $\alpha$ is the heat transfer coefficient to the surroundings, $\mathrm{A}$ is the total surface area of the nanoparticles, and $\Delta T$ is the difference between the temperature of the particles $T_{S}$ and surrounding water $T_{W}$. Thus, the original equation can be rewritten as:

$$
\frac{d T_{S}}{d t}+\frac{\alpha A T_{S}}{\sigma m}=\frac{P+\alpha A T_{W}}{\sigma m}
$$

This is a first order, ordinary differential equation which has the well-known solution

$$
T_{S}=T_{W}+\frac{P\left(1-e^{-\frac{\alpha A t}{\sigma m}}\right)}{\alpha A}
$$

\subsubsection{Heating model results}

Using equation (4), it is possible to estimate the extent of microwave electric field-induced heating in a suspension of nanoparticles, based on the absorbed power, with results shown in Figure 3. First, due to their small size, the metallic germanium particles considered here reach steady-state temperature very rapidly, in less than $1 \mathrm{~ms}$. Secondly, particles can theoretically reach very high temperatures (in the region of hundreds of degrees Celsius), even after accounting for heat losses into the water. Finally, it must be noted, that while values of $10^{12}$ $\mathrm{W} / \mathrm{m}^{3}$ appear to be excessively high, this results in only $0.1 \mathrm{~W}$ of power absorbed by a $10^{9}$ particle/ml suspension.

Figure 3. The temperature reached by $100 \mathrm{~nm}$ spherical nanoparticles in water, with density $5600 \mathrm{~kg} / \mathrm{m}^{3}$, heat capacity $310 \mathrm{~J} \mathrm{~kg}^{-1} \mathrm{~K}^{-1}$ (metallic germanium), as predicted by Equation 4 . The traces represent the heating over time, with 3 different scenarios of the power absorption by the nanoparticles, in $\mathrm{W} / \mathrm{m}^{3}$. The dashed line highlights the boiling point of water. 
The dielectric heating of nanoparticles is only significant in semiconductor particles, such as germanium. To understand this, consider the power dissipated per unit volume within a particle, which is $\sigma E_{1}{ }^{2}$, where $\sigma$ is the electrical conductivity and $E_{1}$ is the internal electric field within each particle (assumed uniform). Thus, metal particles will not dissipate since $E_{1}$ is negligible owing to very effective electrostatic screening; however, neither will low-loss dielectrics dissipate since then $\sigma$ is negligible. In some semiconductors, such as germanium at room temperature, the moderate value of $\sigma$ is sufficiently small so as not to screen $E_{1}$ to very low values, but large enough to cause moderate dissipation (Porch et al., 2013). For nanoparticles made of conductive metals, heating can be treated as zero. The highest possible heating value is $0.1 \mathrm{~W} / \mathrm{m}^{3}$ for an electric field of $1 \mathrm{~V} / \mathrm{m}$ and happens at a conductivity of $0.409 \mathrm{~S} / \mathrm{m}$ (Porch et al., 2013). The closest material to this value used in this study will be germanium. Since the power dissipation is proportional to the square of the electric field magnitude, for heating effects like those shown in Figure 3, a field of the order of $10^{6} \mathrm{~V} / \mathrm{m}$ would be required. Such a field is higher than the typical fields expected in the experimental cavity used here. With a field level of $10^{4} \mathrm{~V} / \mathrm{m}$, as in the current system, the particles will heat by approximately $0.01{ }^{\circ} \mathrm{C}$ over the course of the $3 \mathrm{~ms}$ on-time exposure. This temperature difference will be rapidly equalised by the surrounding water, so overall the heating of the particles with the current experimental conditions can be considered negligible.

We repeat the important fact that direct Ohmic heating of the nanoparticles is negligible in the case of gold owing to the very high electrical conductivities, which give effective screening of the electric field within them. Furthermore, EM scattering is also negligible since all particles are in the limit where the particle size is much less than the microwave wavelength (in our case by a factor of around $10^{5}$ ).

This means that particles will not heat directly using the existing setup. However, at higher field values, heating will be possible for semiconductor particles. The model also highlights the short time required to heat nanoparticles. Nanoparticles heat and reach steady-state temperature in less than $1 \mathrm{~ms}$. This means that with high microwave electric field values, a low duty cycle could be employed (e.g. with $1 \mathrm{~ms}$ on-time).

\subsection{Electric field model using COMSOL}

\subsubsection{Nanoparticles model}

An electric field distribution model of a metallic nanoparticle was generated using COMSOL. A metallic, spherical nanoparticle was simulated in an aqueous solution. The electric field 
within the particle itself is approximately zero due to the screening effect of the conduction band electrons. Quantitatively, the (uniform) electric field within a spherical nanoparticle is:

$$
E_{1}=\frac{3 E_{0}}{2+\varepsilon}
$$

where $E_{0}$ is the applied electric field the particle is subjected to, and $\varepsilon$ is the complex permittivity of the material (Porch et al., 2013). This means that for highly conductive particles, the large imaginary part of the permittivity (owing to the large conductivity value) will drive $E_{1}$ down to be close to zero. Even for a semiconductor particle like germanium, the electric field magnitude within the particle will only be approximately $13 \%$ of that of the applied field. As for the electric field in the space surrounding each particle, it will be focused at the poles in the usual manner; the focussing factor for field amplitude is 3 for a metallic sphere, varying between 1 and 3 for non-metallic particles depending on the size of the relative permittivity $\varepsilon$; this result is predicted theoretically and has been confirmed by COMSOL simulations shown on Figure 4A. By comparison, a particle with the "urchin" shape (though this applies to any shape with sharp angles), is found by simulation to have smaller hotspots focused on the spikes with a much higher focussing factor for the electric field, as large as 7.5 (Figure 4B). Since any action derived from the microwave electric field is expected to be proportional to the field intensity $E^{2}$ (for example, power dissipated) these focussing factors become even more significant.

Figure 4. Results of a 3D COMSOL simulation showing the focusing of an electric field by $100 \mathrm{~nm}$ conducting metallic nanoparticles. Compared to spherical nanoparticles (A), the urchin-shaped nanoparticles (B) generate smaller hotspot zones but with a more strongly focused field, up to a maximum of 7.5 greater than the applied electric field.

When nanoparticles interact with spores, the part of the spore in contact with the nanoparticle will be subjected to higher electric field. In the case of spherical nanoparticles, the enhancement will be small, due to the limited area of contact and low field focusing (Figure 4A). In the case of angled nanoparticles, the field at the contact points will be higher (Figure 4B). In addition, the angled shape will allow the focused field to penetrate deeper into the spore to enhance disruption. In conclusion, the angled nanoparticles generate hotspots with a stronger electric field for a given input microwave power level and are therefore better suited to electric fieldbased spore disruption. 


\subsubsection{Spore model}

The spore is a complex multi-layer structure, with the outer exosporium layer and the innermost core containing some water, it being separated by the multiple spore coat layers and the cell membrane (Setlow, 2007). The main limitation of developing a COMSOL model of a spore is the lack of information on the electric properties of these different spore layers. While values can be estimated, based on the fact that the spore layers contain proteins, lipids and polysaccharides, the results may not be fully representative of the spore itself.

Figure 5. A simple 3D model of multiple spore layers under the effect of an electric field. The electric field is amplified at the simulated dielectric spore coat and membrane layers. The maximum enhancement up more than a factor of 5 in the simulated membrane layer (red), indicating that this layer will see the electric field magnitude, and so the greatest power dissipation if the layer is lossy.

Although the model uses assumptions regarding the exact relative permittivity values and thickness of the layers, the overall result will be the electric field component perpendicular to the membrane layers will be highest in the low permittivity layers (such as the membrane) compared to the aqueous layers and the surrounding layers, irrespective of their arrangement. This is illustrated with the simple model below, the results of which are shown on Figure 6. Consider a stratified material comprising $N$ layers of dielectric material, perpendicular to the plane of which an electric field is applied. We assume that $E_{0}$ is the electric field magnitude in the first and $N^{\text {th }}$ layer, which we will assume is water. Applying the boundary condition for the electric field $\underline{E}$, namely that the component of electric flux density $\underline{D}=\varepsilon_{r} \varepsilon_{0} \underline{E}$ is continuous across each boundary, where $\varepsilon_{r}$ is the complex permittivity of each layer, the electric field magnitude in each layer is then $E=E_{0} /\left|\varepsilon_{r}\right|$. Clearly, the magnitude is reduced in the higher permittivity layers and increased in the lower permittivity layers, the physical origin being the surface density of the polarisation charge density induced in at the boundary between each layer. In Figure 6, we show 9 layers (with 1 and 9 being water), each of random thickness, with layers 2-7 having random complex permittivity values; clearly, layer 6 has the lowest complex permittivity. 
Figure 6. A simple stratified layer module which demonstrates the enhanced electric field magnitude (here in layer 6), supporting the results from Figure 5. The thickness of the layers has no bearing on the calculation of the electric field strength within each, in the limit where the thickness is much less than the microwave wavelength. It only depends on the permittivity of the layers, exactly as one would expect for such quasi-static analysis. Layers 1 to 9 are water, and the other 7 layers have random thickness and complex permittivity values, the lowest value of permittivity being for layer 6 .

An important property of spores can be noted: the low permittivity layers of the spore will, by definition, give rise to the greatest electric field magnitude $|E|$. All field-induced processes will be enhanced in such layers, be they a direct result of the electric field, or a result of heating, where the power dissipated per unit volume is proportional to $\varepsilon_{2}|E|^{2}$, where $\varepsilon_{2}$ is the imaginary (i.e. lossy) part of the complex permittivity. Referring to the spore model, the core is therefore partially shielded from the electric field, and the model suggests the field strength at this point is only $75 \%$ of that of the applied electric field. By contrast, the dielectric layers will have higher field values, rising to more than a factor of 5 in the sections of the membrane layer perpendicular to the applied field direction.

The low water content layers (spore coat and membrane) are dielectrics separating two conductive layers: the aqueous solution containing the spores, and the spore core. This means they can function effectively as capacitors, storing electrical energy (and, indeed, also dissipating energy as heat if the layer allows) when exposed to microwaves. Such a build-up of energy could have consequences on the spore, such as structure degradation, which could lead to DNA release.

In conclusion, the spore model provides a possible insight into the disruption of spores. The spore surface is well hydrated and therefore with high relative permittivity closer to that of water. This layer will be subjected to a lower electric field and thus is unlikely to be damaged in a manner that will be visible under an SEM (scanning electron microscope). By contrast, the inner dehydrated layers of the coat and membrane will experience a higher level of electric field, so may be disrupted due to realignment of charged groups leading to membrane damage and subsequent DNA release.

\subsection{Validation of the model}


The model described above predicts nanoparticles are able to focus the microwave electric field in the vicinity of spores. To validate this model experimentally, spores were exposed to microwaves in the presence of different nanoparticle suspensions in order to compare the observations with the model's predictions.

\subsubsection{DNA release by microwaved spores}

To evaluate the potential additional contribution of nanoparticles, the concentration of microwave-mediated DNA release in the absence of nanoparticles was first determined. As can be seen in Figure 7, the release of DNA was very small, with the highest value of $35 \mathrm{pg} / \mu \mathrm{l}$. This level of DNA was not statistically significant ( $p=0.35$ with a hypothesis of no release).

Figure 7. ssDNA release from $C$. difficile spores following a $0.3 \%$ duty cycle, 30 second microwave exposure ( $\mathrm{n}=3$ ). Overall, the change in the ssDNA signal was not statistically significant (compared to zero release). Error bars indicate the standard deviation of results.

Next, the effect of different nanoparticles on the microwave-induced DNA release was determined. The results in Figure 8 show an increase in DNA release in the presence of nanoparticles for each test combination.

Figure 8. The effect of different nanoparticle suspensions on DNA release following microwave exposure. The results show the change in DNA release, compared to a control sample which has been microwaved without nanoparticles $(n=3$, see Figure 6). Stars indicate the degree of significance in the deviation from all the groups, using the Bonferroni post-test; one star for $\mathrm{p}<0.05,2$ stars for $\mathrm{p}<0.01$, three stars for $\mathrm{p}<0.001$. Error bars indicate the standard deviation of results.

The degree of DNA release following microwave exposure varied depending on the clinical isolate and nanoparticle used. Overall the differences in the DNA release were statistically significant (ANOVA $\mathrm{p}<0.0001$ ), demonstrating that nanoparticles improve spore disruption compared to the samples without nanoparticles (Figure 7). In the case of the R20291 strain, all nanoparticles had a statistically significant increase in DNA levels. In the case of the DS1813 strain, all nanoparticles except the $100 \mathrm{~nm}$ gold and $30 \mathrm{~nm}$ gold had a statistically significant effect on increasing DNA release. Regarding the CD630 strain and DS1748 strains, there were increases in the DNA release, but these were deemed to be statistically insignificant. 


\subsubsection{Characterisation of spores exposed to nanoparticles}

In order to better understand the reasons of the increased DNA release in the presence of nanoparticles, the microwaved spore suspensions from the samples that were used in the previous section were examined by using SEM for any visual signs of spore disruption.

As can be seen from Figure 9A and Figure 9B, microwave exposure in the presence of $100 \mathrm{~nm}$ spherical gold nanoparticles had no visible effect on the spore architectures. Similar results were observed in all four clinical isolates, including R20291 (Figure 9B), where the $100 \mathrm{~nm}$ gold nanoparticles have been shown to have a statistically significant increase in DNA release.

Figure 9. SEM of $C$. difficile spores exposed to microwaves in the presence of spherical gold nanoparticles. A: DS1748 strain, $100 \mathrm{~nm}$ particles. B: R20291strain, $100 \mathrm{~nm}$ particles. C: DS1748 strain, $30 \mathrm{~nm}$ particles. D: DS1748, $90 \mathrm{~nm}$ urchin-shaped particles. The magnification was $\times 25,000$. These images are representative of 10 fields of view for each sample.

Neither the $30 \mathrm{~nm}$ spherical (Figure 9C) or $90 \mathrm{~nm}$ urchin gold nanoparticles (Figure 9D) had any visible effect on the surface architectures of the spores of all four clinical isolates. By contrast, spores microwaved in the presence of germanium nanoparticles were heavily disrupted, with collapsed and partially collapsed spores observed. Similar disruption was seen in all four clinical isolates, even though it did not lead to a statistically significant increase in DNA release from the DS1748 and CD630 strains.

Figure 10. SEM of $C$. difficile spores of the DS1 748 strain spores exposed to microwaves in the presence of $30 \mathrm{~nm}$ germanium nanoparticles. The magnification was $\times 25,000$. The spores appear damaged and collapsed in on themselves (highlighted), in contrast to the intact spores with other nanoparticles (Figure 9). These images are representative of 10 fields of view.

Overall, these results show that DNA release from spores microwaved in the presence of gold spherical and urchin-shaped nanoparticles is not associated with any visible structural damage of the spores, indicating that the disruption mechanism is non-destructive in nature. The method of using germanium nanoparticles is associated with significant spore damage, suggesting a different mechanism of action. 


\section{Discussion}

The heating simulation of the individual nanoparticles suggests that in aqueous solutions semiconductor particles theoretically reach very high temperatures, providing there is sufficient electric field (of order of $10^{6} \mathrm{~V} / \mathrm{m}$ ). However, due to the high surface area to volume ratio, this is likely to be very inefficient and will only work with semiconductor materials like germanium. Under the existing setup and an electric field of order of $10^{4} \mathrm{~V} / \mathrm{m}$, the heating will be of the order of $0.01{ }^{\circ} \mathrm{C}$ per pulse and will be completely negated by the surrounding aqueous solution.

The electric field model in COMSOL indicates that nanoparticles will focus the electric field, as expected theoretically, and angled nanoparticles (i.e. urchin-shaped), will create small zones of very focused field, while spherical nanoparticles create larger high field areas, but with less field focusing. This means that when a nanoparticle comes in contact with the spore, only a very small area (and thus point volume) will be subjected to the higher electric fields. With angled nanoparticles, the field at the contact points will be higher and therefore a larger proportion of the spore will be exposed to these high fields. As was previously stated, the electric field effects scale quadratically since it is the field intensity that is important, so a field focusing factor of 2 will have 4-fold enhanced effect. Likewise, with a field focusing factor of 7.5 , as was predicted for the urchin model, the electric field effects will be 56-fold enhanced. In addition, the urchin shape has multiple focused field hotspots, allowing each particle to affect several locations on the spore simultaneously. In contrast, a spherical nanoparticle has only two hotspots at opposite poles, making it much less effective.

The electric field model of the spore indicates that the electric field is focused in the membrane and spore coat, but not on the spore surface. This field enhancement affects most of the membrane and coat layer, except for the two polar areas of the spores which are aligned with the electric field. This presents a potential model for nanoparticle-mediated DNA release enhancement. Without nanoparticles, the outermost layer is intact, therefore DNA release is limited even if the inner coat and membrane layers are heavily disrupted. Our hypothesis is that nanoparticles create small zones of disruption in the outer spore layers due to the electric field hotspots, giving a route for DNA to the be released. Due to the size of these hotspots, they may not be visible under SEM and the spore will still appear intact. 
The results from these preliminary experiments show that microwave-mediated DNA release from spores of the R20291 and DS1813 strains can be enhanced by the addition of nanoparticles, particularly the gold urchin-shaped nanoparticles and germanium nanoparticles.

To determine the effect of shape and size on DNA release, the interaction of R20291 and DS1813 spores with gold spherical (30 and $100 \mathrm{~nm}$ ) and urchin shaped (90 nm) nanoparticles was examined. A different pattern of response for the two strains was seen with regards to DNA release, which suggested that spore structure may have a major impact on how they interact with nanoparticles. Only the urchin-shaped nanoparticles caused a significant increase in DNA release from both strains, which supports the data from the electric field COMSOL model and the prediction that the hotspots generated by the urchin-shaped nanoparticles enhance DNA release. While the urchin nanoparticles mediated DNA release from both strains, it was less effective than spherical gold nanoparticles for R20291 spores, suggesting that factors other than shape may be important.

Finally, the effect of germanium nanoparticles was also analysed. Germanium nanoparticles increased spore DNA release in the DS1813 and R20291 strains and were associated with visible destructive spore disruption. This disruption, however, did not correlate with significantly higher DNA release compared with the non-destructive disruption of gold nanoparticles. The visual signs of disruption do indicate a different mechanism may be responsible for the spore disruption. The modelling results rule out a heating-based effect, as the electric field strength in in the cavity is insufficient to cause any heating in the test conditions used. It may, however, be due to a chemical effect from the surfactant. The germanium nanoparticles came from a different supplier (US-Nano) to the gold nanoparticles (Sigma-Aldrich), and were supplied with a proprietary surfactant. This means the attachment of these nanoparticles to spores could be different to that of other nanoparticles dispersions. The irregular shape of the nanoparticle could also be a factor, generating hotspots similar to the urchin-shaped nanoparticles.

The CD630 strain was the least affected. Only the $90 \mathrm{~nm}$ urchins had an average improvement of over $50 \mathrm{~nm} / \mu \mathrm{l}$ of DNA release. A possible explanation is a low DNA content in the outer spore surface. This strain lacks the "pineapple" outer layer of the DS1813 and DS1748, or the large baggy outer layer of the R20291 strain. If the DNA is trapped in the spore layer as hypothesised, there may simply be less areas for the DNA to be trapped in the CD630 strain compared to others. 
Attachment of nanoparticles to spores due to different surface properties may also play a major role in spore disruption. This attachment could potentially be enhanced by functionalisation. The nanoparticles used in the study were citrate-stabilised, and therefore had a partial negative charge on the surface (Zhao et al., 2013). This could cause the nanoparticles to repel each other, resulting in poor interaction with hydrophobic spores such as the DS1748 and DS1813 strains. Functionalised nanoparticles could contain positive charge groups on the surface and therefore bind more easily.

One of the limitations preventing higher statistical significance of experimental results for DNA release was the Qubit assay itself. The issues with spore clumping and nanoparticle clumping did lead to a larger variation in results, which led to higher $\mathrm{p}$ values.

Based on the electric field model, due to water having a very high dielectric loss for a 2.45 $\mathrm{GHz}$ frequency, it inevitably absorbs much of the power that goes into the sample. This means that water interferes with spore disruption and the power absorbed by the water is effectively wasted. For a more effective spore disruption it may be better to redesign the system to work with dry samples, where water is only added after microwaving, as a solvent for the DNA. Such a system will need much less power, as the sample mass and total heat capacity will be much smaller. Doing this also eliminates the sources of systematic error from dispersants as well as nanoparticle and spore clumping interference in Qubit or other fluorescence assays.

In conclusion, nanoparticles were found to significantly enhance spore disruption, with gold urchin shaped and germanium irregular shaped particles showing the greatest effect. The model of nanoparticle and spore interactions indicates that the urchin and irregular shaped nanoparticles produce localised areas of concentrated field on the spore surface where spore disruption enhanced. This data can be used to enhance the design of microwave-based diagnostic systems for bacterial spores in a rapid and specific manner.

\section{Acknowledgments}

We would like to thank Dr. Emmanuel Brousseau from the Cardiff School of Engineering, Cardiff University for allowing the use of the electron microscopy facility and technical assistance with its operation. 


\section{References}

Acharya, D., Singha, K. M., Pandey, P., Mohanta, B., Rajkumari, J., and Singha, L. P. (2018). Shape dependent physical mutilation and lethal effects of silver nanoparticles on bacteria. Scientific Reports, 8(1), 201.

Aslan, K., Previte, M. J. R., Zhang, Y., Gallagher, T., Baillie, L., and Geddes (2008). Extraction and detection of DNA from Bacillus anthracis spores and the vegetative cells within 1 min. Analytical Chemistry, 80, 4125-4132.

Aslan, K., Baillie, L. and Geddes, C. D. (2010) Ultra fast and sensitive detection of biological threat agents using microwaves, nanoparticles and luminescence. Journal of Medical Chemical, Biological and Radiological Defense, 8, 1-21.

Barbut, F., Braun, M., Burghoffer, B., Lalande, V., and Eckert, C. (2009). Rapid detection of toxigenic strains of Clostridium difficile in diarrheal stools by real-time PCR. Journal of Clinical Microbiology, 47(4), 1276-1277.

Bibi, F., Villain, M., Guillaume, C., Sorli, B., and Gontard, N. (2016). A review: origins of the dielectric properties of proteins and potential development as bio-sensors. Sensors, 16(8), 1232.

Celandroni, F., Longo, I., Tosoratti, N., Giannessi, F., Ghelardi, E., Salvetti, and S., Baggiani, A., Senesi, S. (2004) Effect of microwave radiation on Bacillus subtilis spores. Journal of Applied Microbiology 97 (6) 1220-1227

CRDN (Public Health England) Clostridium difficile Ribotyping Network (CDRN) for England and Northern Ireland 2011 - 13 Report.

Đapa, T., Leuzzi, R., Ng, Y. K., Baban, S. T., Adamo, R., Kuehne, S. A., Unnikrishnan, M. (2013). Multiple Factors Modulate Biofilm Formation by the Anaerobic Pathogen Clostridium difficile. Journal of Bacteriology, 195(3), 545-555.

Dilger, J. P., McLaughlin, S. G., McIntosh, T. J., and Simon, S. A. (1979). The dielectric constant of phospholipid bilayers and the permeability of membranes to ions. Science, 206(4423), 1196-1198. 
Fordtran, J. S. (2006). Colitis due to Clostridium difficile toxins: underdiagnosed, highly virulent, and nosocomial. In Baylor University Medical Center. Proceedings (Vol. 19, No. 1, p. 3). Baylor University Medical Center.

Joshi, L. T., Mali, B. L., Geddes, C. D., and Baillie, L. (2014). Extraction and sensitive detection of toxins $\mathrm{A}$ and $\mathrm{B}$ from the human pathogen Clostridium difficile in 40 seconds using microwave-accelerated metal-enhanced fluorescence. PloS one, 9(8), e104334.

Kim, S.Y., Shin S.J., Song, C.H., Jo, E.K., Kim, H.J., and Park, J.K. (2009) Destruction of Bacillus licheniformis spores by microwave irradiation Journal of Applied Microbiology, 106 (3) $877-885$

Kim, K., Seo, T., Sim, K., and Kwon, Y. (2016). Magnetic nanoparticle-assisted microwave hyperthermia using an active integrated heat applicator. IEEE Transactions on Microwave Theory and Techniques, 64(7), 2184-2197.

Lawley, T. D., Croucher, N. J., Yu, L., Clare, S., Sebaihia, M., Goulding, D., Dougan, G. (2009). Proteomic and Genomic Characterization of Highly Infectious Clostridium difficile 630 Spores Journal of Bacteriology, 191(17), 5377-5386.

Lyu, C., Wang, J., Powell-Palm, M., and Rubinsky, B. (2018). Simultaneous electroporation and dielectrophoresis in non-electrolytic micro/nano-electroporation. Scientific Reports, $8(1)$, 2481.

Office of National Statistics (UK) Deaths Involving Clostridium difficile, England and Wales, 2012 http://www.ons.gov.uk/ons/dcp171778_323989.pdf accessed 01/06/2018

Paredes-Sabja, D., Cofre-Araneda, G., Brito-Silva, C., Pizarro-Guajardo, M., \& Sarker, M. R. (2012). Clostridium difficile spore-macrophage interactions: spore survival. PloS one, 7(8), e43635.

Pearce, J. A., Cook, J. R., and Emelianov, S. Y. (2010). Ferrimagnetic nanoparticles enhance microwave heating for tumor hyperthermia therapy. In Engineering in Medicine and Biology Society (EMBioC), 2010 Annual International Conference of the IEEE (pp. 2751-2754). IEEE.

Pellerin, C. (1994). Alternatives to Incineration: There's More Than One Way to Remediate. Environmental Health Perspectives, 102(10), 840-845. 
Pizarro-Guajardo, M., Calderón-Romero, P., Castro-Córdova, P., Mora-Uribe, P., \& Paredes-Sabja, D. (2016). Ultrastructural Variability of the Exosporium Layer of Clostridium difficile spores. Applied and environmental microbiology, AEM-03410.

Porch, A., Slocombe, D., and Edwards, P.P. (2013) Microwave absorption in powders of small conducting particles for heating applications. Physical Chemistry Chemical Physics 15(8):2757-63

Setlow, P. (2007) I will survive: DNA protection in bacterial spores. Trends in Microbiology $15(4), 172-180$

Sorg, J. A. and Sonenshein, A. L. (2008) Bile salts and glycine as cogerminants for Clostridium difficile spores? Journal of Bacteriology, 190, 2505-2512.

Sorg, J. A., and Sonenshein, A. L. (2010). Inhibiting the Initiation of Clostridium difficile Spore Germination using Analogs of Chenodeoxycholic Acid, a Bile Acid. Journal of Bacteriology, 192(19), 4983-4990.

Tian, P. (2010). Computational protein design, from single domain soluble proteins to membrane proteins. Chemical Society Reviews, 39(6), 2071-2082.

Vaid, A. and Bishop, A. H. (1998). The destruction by microwave radiation of bacterial endospores and amplification of the released DNA. Journal of Applied Microbiology, 85, 115122

Williams, C. F., Geroni, G. M., Pirog, A., Lloyd, D., Lees, J., and Porch, A. (2016). The separated electric and magnetic field responses of luminescent bacteria exposed to pulsed microwave irradiation. Applied Physics Letters, 109(9), 093701. 


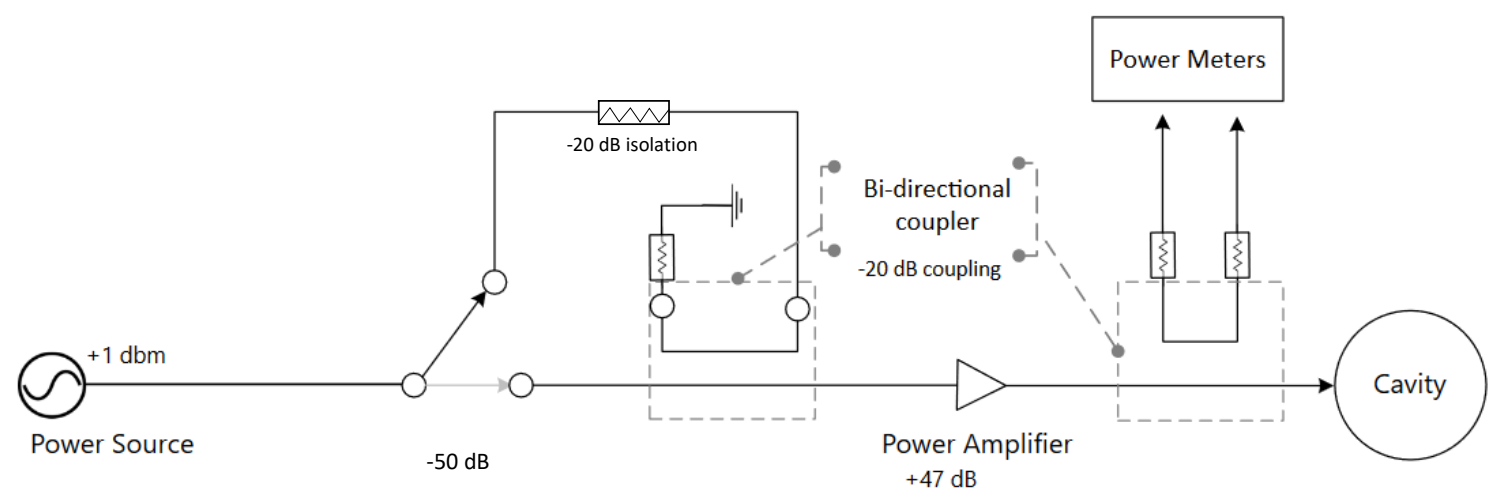




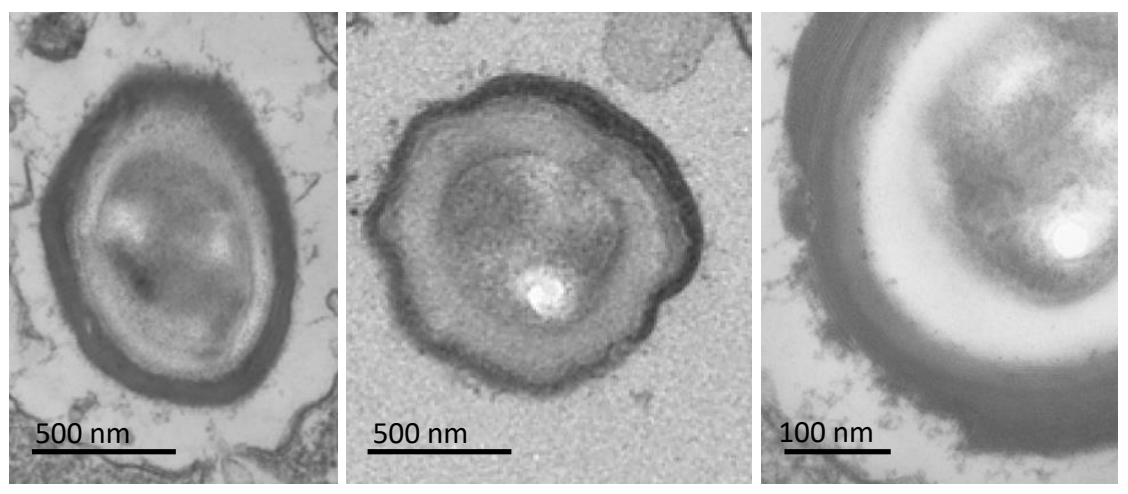




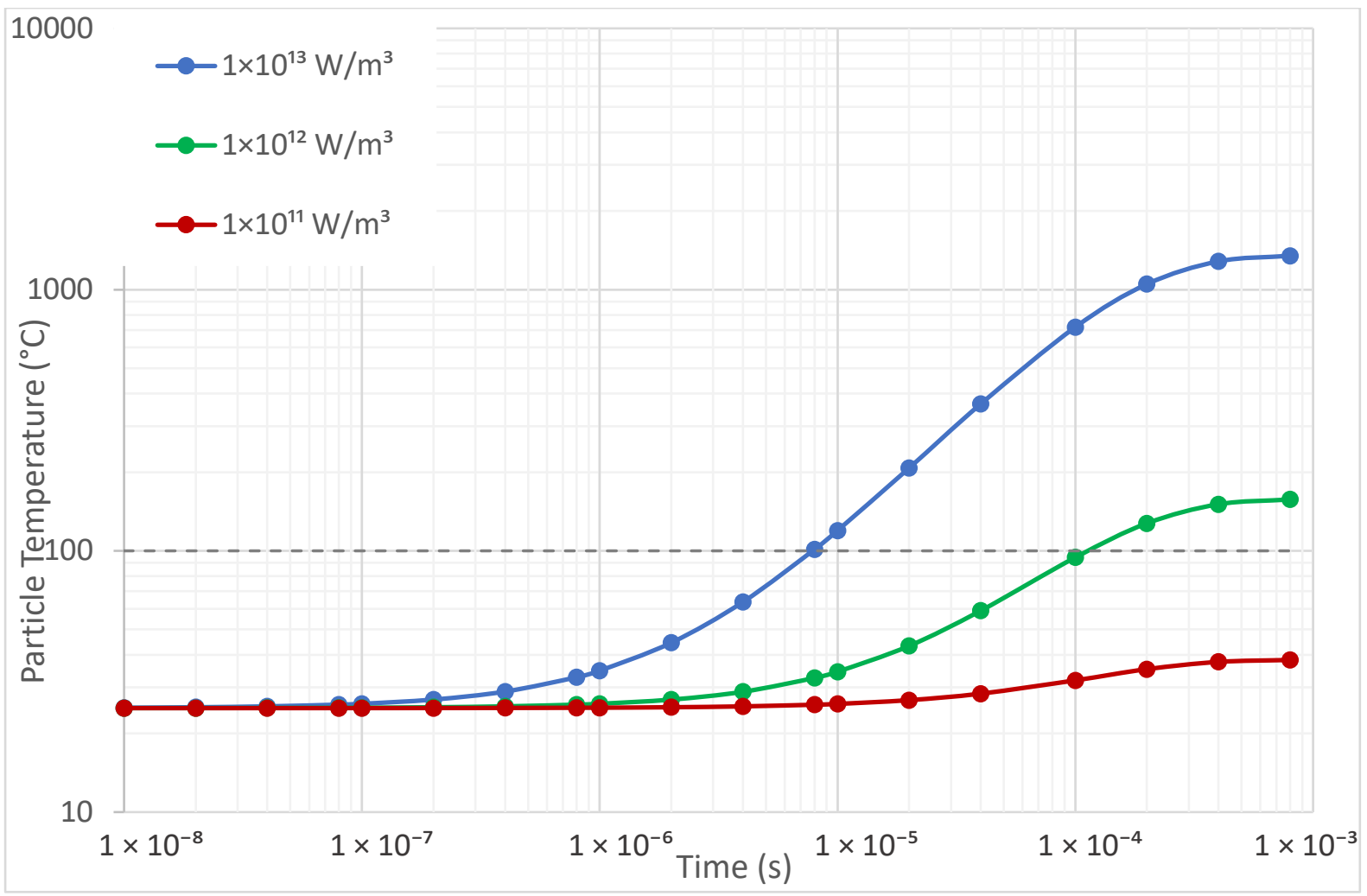




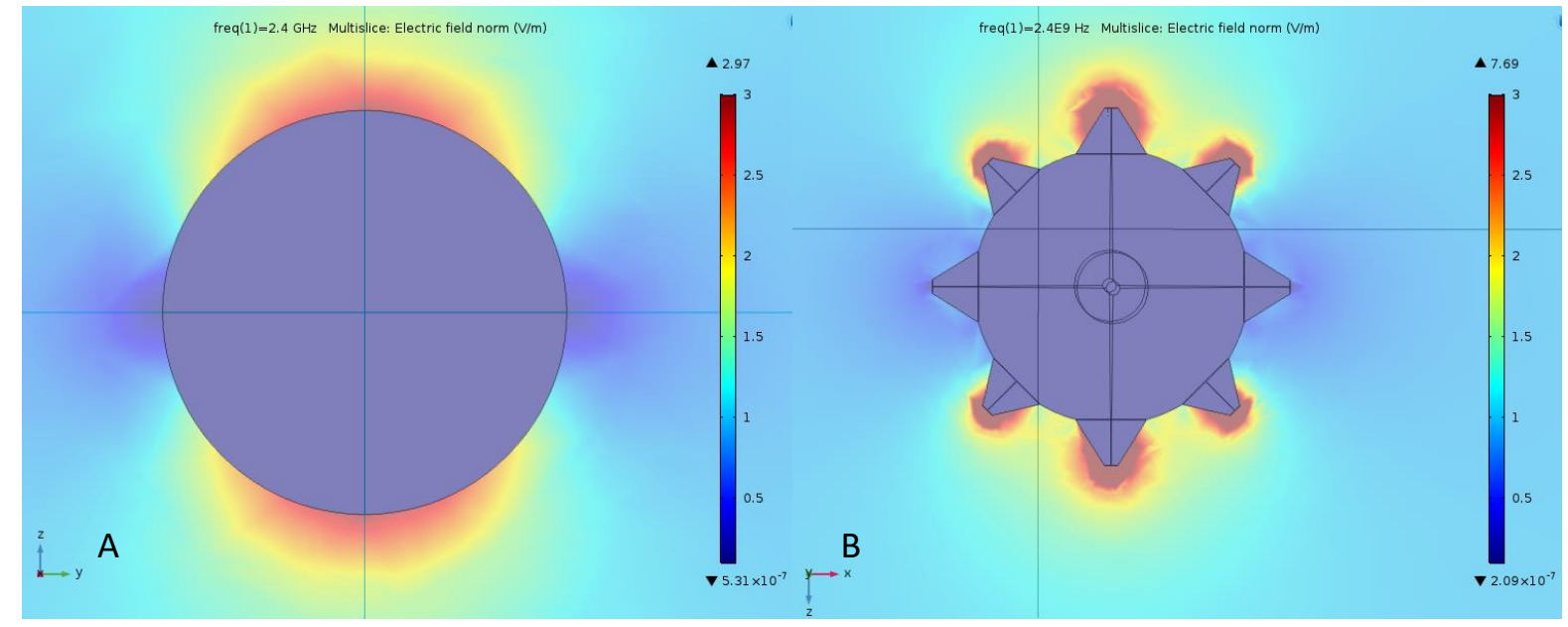





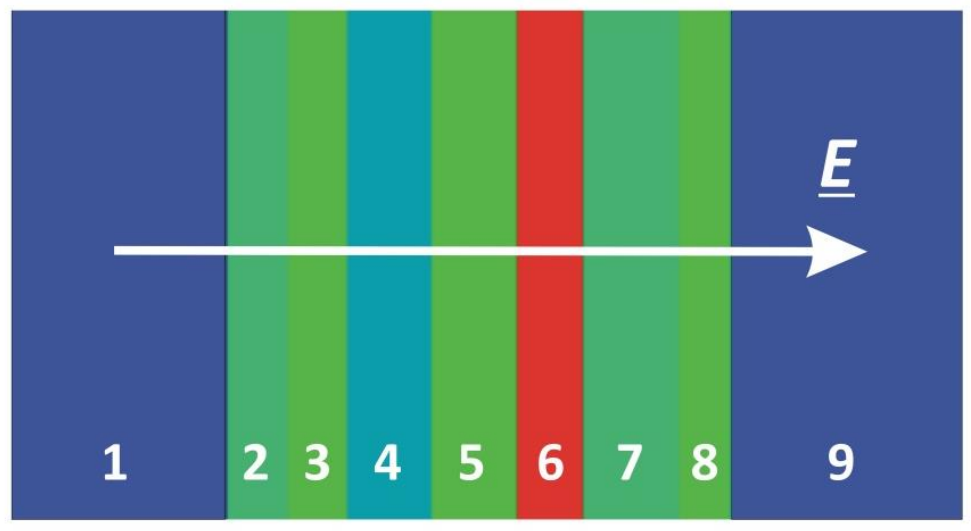


100.0

75.0

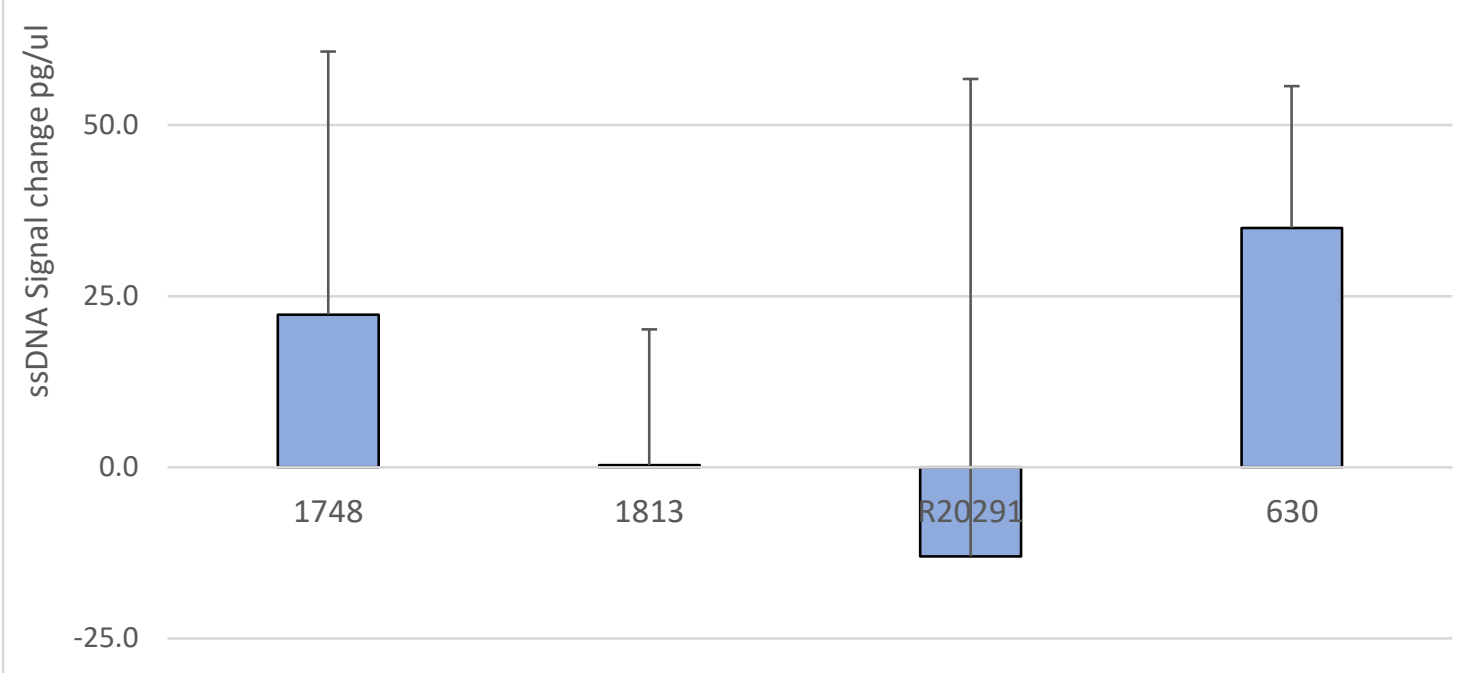




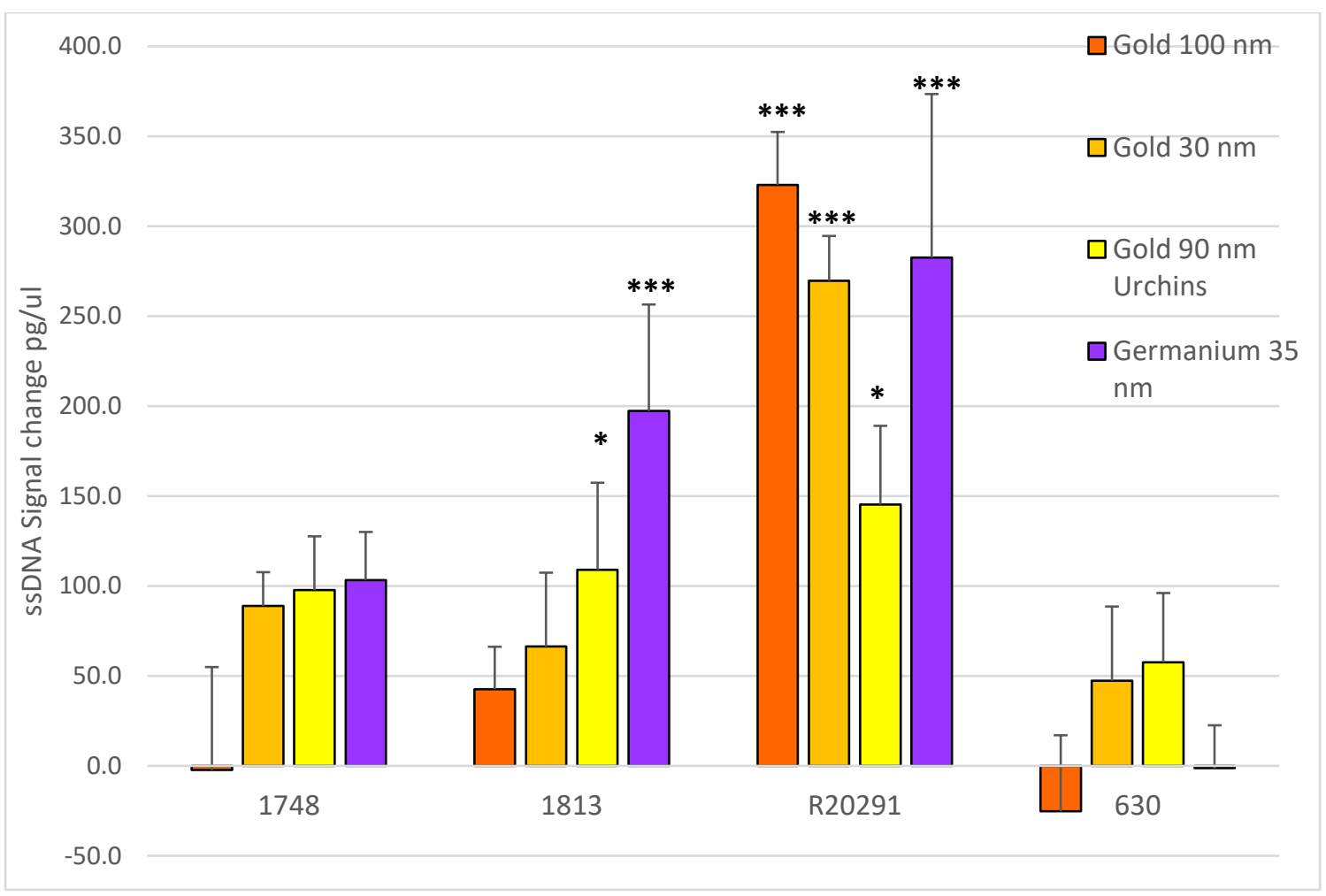




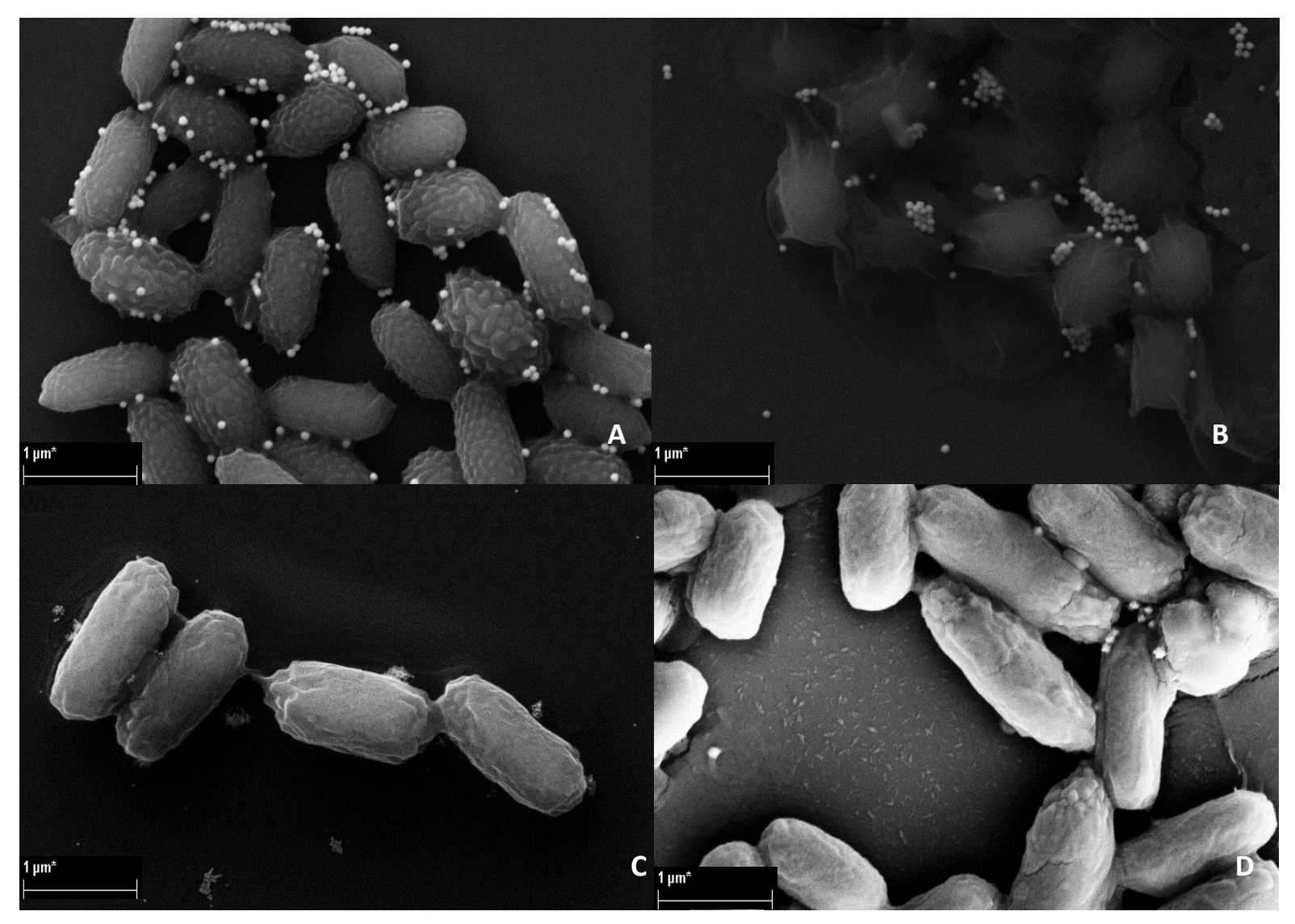




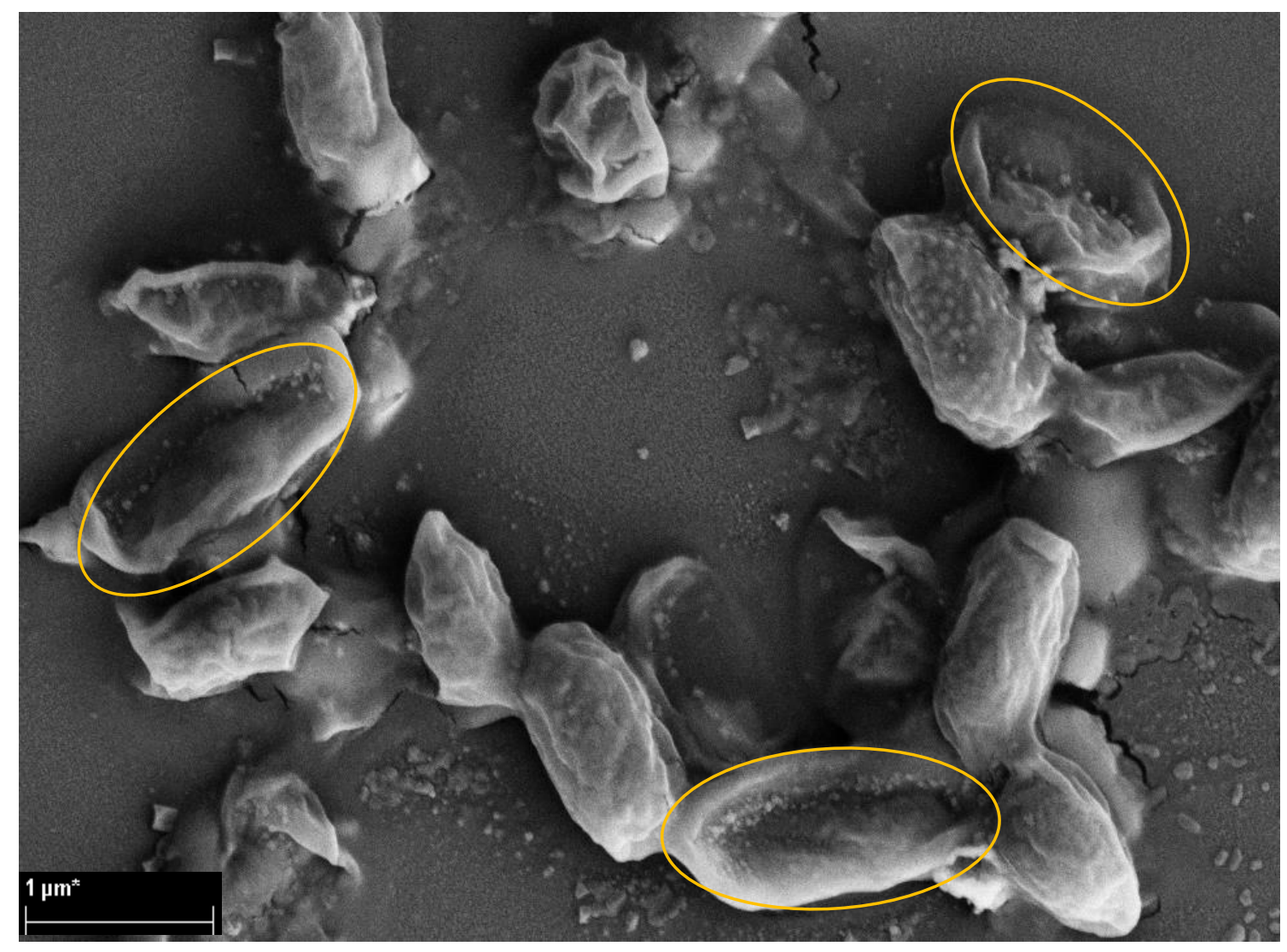

